\title{
Is desynchrony tolerance adaptable in the perceptual organization of speech?
}

\author{
Robert E. Remez, Daria F. Ferro, Kathryn R. Dubowski, \\ Judith Meer, Robin S. Broder, ANd Morgana L. DavidS \\ Barnard College, Columbia University, New York, New York
}

\begin{abstract}
Speech signal components that are desynchronized from the veridical temporal pattern lose intelligibility. In contrast, audiovisual presentations with large desynchrony in visible and audible speech streams are perceived without loss of integration. Under such conditions, the limit of desynchrony that permits audiovisual integration is also adaptable. A new project directly investigated the potential for adaptation to consistent desynchrony with unimodal auditory sine-wave speech. Listeners transcribed sentences that are highly intelligible, with veridical temporal properties. Desynchronized variants were created by leading or lagging the tone analog of the second formant relative to the rest of the tones composing the sentences, in 50-msec steps, ranging from 250-msec lead to 250 -msec lag. In blocked trials, listeners only tolerated desynchronies $<50 \mathrm{msec}$, and exhibited no gain in intelligibility to consistent desynchrony. Unimodal auditory and bimodal audiovisual forms of perceptual integration evidently exhibit different temporal characteristics, an indication of distinct perceptual functions.
\end{abstract}

How do sensory samples form integrated perceptual streams? In speech perception, two aspects of sensory integration are prominent. In one, sensitivity to the pattern of modulation of a heterogeneous variety of acoustic elements promotes perceptual coherence (Elliott \& Theunissen, 2009; Remez, Rubin, Berns, Pardo, \& Lang, 1994), in contrast to piecemeal aggregation of acoustic elements (Bregman, 1990; Darwin, 1997). In another, the perceptual organization of speech is ineluctably multimodal, observed in studies showing that speech is protected from masking when a talker is visible as well as audible (Sumby \& Pollack, 1954) and in studies in which a visible face influences perception even when audible speech occurs in the clear (McGurk \& MacDonald, 1976). This observation of sensory merger across modalities has been extended to the claim that sensitivity to coherent modulation occurs in a common amodal metric independent of sensory modality (Fowler, 2004; Green, 1998; Rosenblum, 2005; cf. Bernstein, Auer, \& Moore, 2004; Massaro \& Stork, 1998). Although a principle of perceptual organization that applies within and across sensory modalities is appealing in its simplicity and breadth, this notion is difficult to reconcile with recent reports about speech.

Several studies of auditory perceptual organization have calibrated perceptual integration of an acoustic speech signal that was manipulated to depart parametrically from a temporally veridical pattern. In one, noise-band vocoded versions of spoken sentences were used (Fu \& Galvin, 2001), and in another, sine-wave replicas of spoken sentences were used (Remez, Ferro, Wissig, \& Landau, 2008). Despite their different treatments of the speech spectrum, each of these methods presents a signal that differs greatly from the harmonically rich spectrum of natural speech. In consequence, intelligibility is likely to depend on sensory integration of the desynchronized components, and less likely to reflect phonetic impressions evoked by individual acoustic components that do not cohere. In these studies, whether desynchrony was applied to adjacent noise bands or to tone analogs of the formants, perceptual integration was lost with small departures from temporal veridicality.

Both of these acoustic treatments of the speech spectrum eliminate local pockets of synchrony in the frequency domain, the first by imposing a kind of spectral blur within frequency bands $1 \mathrm{kHz}$ wide, the second by imposing a sparse distribution of energy across four time-varying sinusoids. In the case of noise-band sentences, performance fell almost by half at a desynchrony of $40 \mathrm{msec}$, and by $80 \%$ of the performance range at a desynchrony of $80 \mathrm{msec}$. In the sine-wave case, shown by the dashed line in Figure 1, performance fell by half at a desynchrony of $50 \mathrm{msec}$, and by $95 \%$ of the performance range at a desynchrony of 100 msec.

Although both projects reported a narrow window of integration, their estimates differed from the findings of studies indicating a more leisurely pace of auditory integration, noted as 3-8 Hz (Saberi \& Perrott, 1999) or as the duration of a syllable (Greenberg \& Arai, 1998; Greenberg, Carvey, Hitchcock, \& Chang, 2003), which in English is $180 \mathrm{msec}$ on average (see also Poeppel, 2003). Across this set of discrepant estimates of integration, though, the physical treatment of the speech signal determined whether or not the performance measure exhibited

R. E. Remez, remez@columbia.edu 


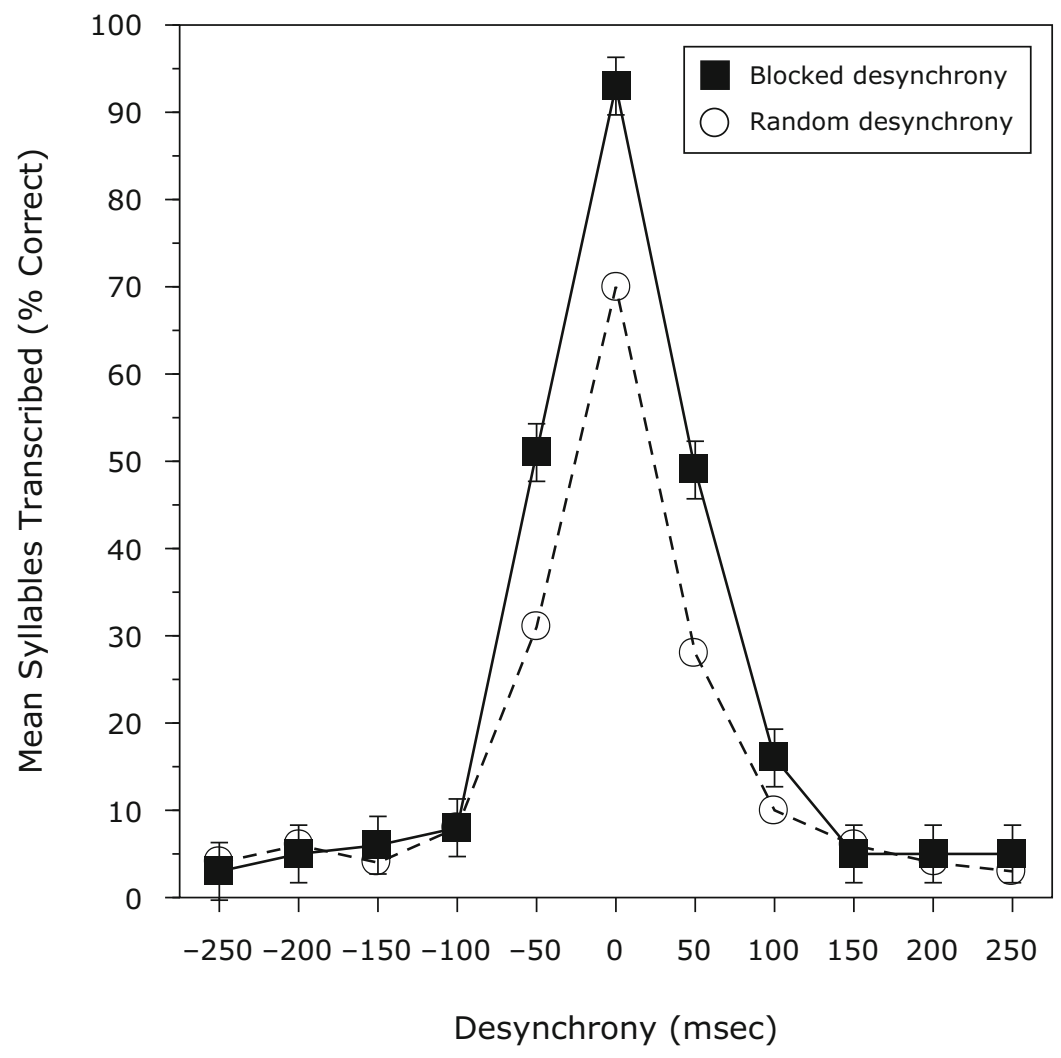

Figure 1. Performance estimates of desynchrony tolerance for blocked and random presentations of asynchrony. Square filled bullets $(\square)$, solid line: Measures of the present test, which presented test items blocked by asynchrony; error bars show the $\mathbf{9 5 \%}$ confidence intervals. Round open bullets $(O)$, dashed line: Results of the tests of Remez et al. (2008), in which asynchrony was random from trial to trial. Desynchrony is the parameter of the $x$-axis, with the veridical temporal relation between the tone analog of the second formant set to 0 msec, and variants departing in 50-msec steps from that value.

greater or lesser tolerance of desynchrony. The projects reporting a longer span of integration used speech samples for which temporal distortions were imposed on spectrally rich acoustic constituents. This method preserved local synchrony and the phonetic properties that can be evoked without spectrotemporal sensory integration. Accordingly, the measures of intelligibility reflected the projection of spectrally rich components into phonetic properties, even when integration was lost or compromised due to temporal distortion. In contrast, the noise-band and sine-wave methods were effective phonetically only when integration occurred, providing a purer measure of integration by neutralizing the contribution to intelligibility of unintegrated signal constituents.

The finer-grain estimates of temporal integration obtained with noise-band vocoded speech and sine-wave speech derive from acoustic treatments in which integration failure could not be offset by the effects of local synchrony, nor by cognitive compensation (see Silipo, Greenberg, \& Arai, 1999). Although this account might explain the preceding reports, the estimate based on sine-wave speech also hints at the greater integrative flexibility that these measures indicate.

\section{Is Desynchrony Tolerance Adaptable?}

A potentially useful clue about the causes of the difference in integration estimates $-50 \mathrm{msec}$ versus $180 \mathrm{msec}$ - is provided by a discrepancy in the intelligibility of 15 temporally veridical sine-wave sentences assayed in two conditions by Remez et al. (2008). In a pilot test to calibrate a baseline of intelligibility, performance was $98 \%$ correct. But in an experimental procedure to calibrate desynchrony tolerance, performance on the same test items was $72 \%$ correct. In the pilot, the sentences were presented as a block of temporally veridical sine-wave replicas. In the experimental procedure, however, listeners transcribed a sequence of sentences differing in the temporal alignment of the tone analog of the second formant. Intelligibility of a sine-wave sentence was boosted when it occurred amid a sequence of temporally veridical sentences, and hampered when it occurred among temporally distorted items.

Prior studies had shown that the intelligibility of sinewave speech depended absolutely on the presence of tone analogs of both the first and second formants (Remez, Rubin, Pisoni, \& Carrell, 1981). Disrupting the veridical temporal alignment of the second formant tone and the 
remaining tones offered, therefore, a test of perceptual integration in which intelligibility could legitimately serve as an estimate of integration. The degree of desynchrony varied unpredictably from trial to trial, from 250 -msec lead to $250-\mathrm{msec}$ lag in $50-\mathrm{msec}$ steps, and as a result, a sine-wave sentence with temporally veridical properties occurred unpredictably on only one or two trials within a block. Whether consistent desynchrony promoted good performance or unpredictable desynchrony harmed performance, this susceptibility to variation in temporal distortion suggests that the performance estimates of desynchrony tolerance provided by Remez et al. (2008) might have underestimated the adaptability and range of sensory integration.

The present project sought a measure of adaptable tolerance of desynchrony in auditory perceptual organization. Here, we report results of a procedure of consistent presentation in which a set of trials challenged each listener with the same departure from veridical temporal properties. In all cases, the tone analog of the second formant of the sentence was desynchronized from the rest of the tone complex in steps of $50 \mathrm{msec}$, from 250 -msec lead to 250 -msec lag. A block of temporally veridical sine-wave sentences was also included among these conditions, a replication of the pilot test of Remez et al. (2008). The results showed gains in intelligibility relative to continually varying desynchrony only at temporal offsets $\leq 50 \mathrm{msec}$ and no evidence of intelligibility gains at greater degrees of temporal distortion.

\section{METHOD}

\section{Acoustic Test Material}

Fifteen four-tone sine-wave sentences (see the Appendix) and the desynchronized variants from Remez et al. (2008) were used as test items. The sentences were drawn from the phonemically balanced IEEE set (Egan, 1948) and the Speech Perception in Noise set (Kalikow, Stevens, \& Elliott, 1977). Natural models for sinewave synthesis had been spoken by a male talker (R.E.R.) seated in a sound-attenuating chamber. The natural utterances were sampled at $22.05 \mathrm{kHz}$ and equated for amplitude, and spectral analyses had been performed to create frequency and amplitude parameters for a synthetic replica of each sentence composed of four time-varying sinusoids. Each sinusoid replicated the pattern of frequency and amplitude changes of one of the four lowest frequency vocal resonances, as well as bursts, frictions, and murmurs. The acoustic analyses were performed interactively by tracing the physical features of the natural samples on a spectrographic display. The four timevarying sinusoids composing a sentence were represented in synthesis parameters at a grain of $10 \mathrm{msec}$. Waveforms were calculated with 16-bit amplitude resolution at a sampling rate of $44.1 \mathrm{kHz}$ and were stored in sampled data format (Rubin, 1980).

Each of the 15 sentences was synthesized in its veridical temporal pattern, nominally 0 -msec desynchrony, and in desynchronized versions created by offsetting the second formant tone. Ten desynchronized versions of each sentence were synthesized with temporal offsets, at $50-\mathrm{msec}$ steps. These ranged from 50 to $250 \mathrm{msec}$ of lead and from 50 to $250 \mathrm{msec}$ of lag of the analog of the second formant relative to the temporally unaltered remainder of the sinewave sentence pattern. Test items were stored in sampled data format, and test sequences were transferred to compact disc. With listeners seated in a sound-attenuating chamber, test items were delivered via Beyerdynamic DT770 headphones at a nominal level of $68 \mathrm{~dB}$ SPL.

\section{Participants}

Ninety-nine listeners were tested, assigned randomly to one of the 11 desynchrony conditions. They were drawn from the undergraduate population of Columbia University, and each received credit toward a course requirement in exchange for their participation. Each was a native speaker of English and reported normal hearing at the time of testing. To assess the susceptibility of each listener to sinewave speech, a brief transcription pretest of six intact, novel sinewave sentences was used, and inclusion in the study was based on performance on this test. Eleven listeners were excluded from the data set for declining or failing to transcribe these preliminary sentences, leaving 8 in each of the 11 desynchrony conditions.

\section{Procedure}

Eleven test sessions were conducted. Within a session, 15 sinewave sentences were used, each exhibiting a consistent degree of desynchrony in the second formant tone. The order of presentation of sentences was random. A single trial consisted of five repetitions of a sine-wave sentence, during which a listener transcribed it.

\section{RESULTS}

Each listener contributed 15 measures, the percent of syllables transcribed correctly, 1 for each sentence within a test block. The performance level at each of the 11 asynchronies was the average of these transcriptions; group averages therefore represent the mean of 120 points for each value of desynchrony. A one-way repeated measures ANOVA performed on the transcription measures for the factor desynchrony revealed a significant effect of desynchrony on performance level $[F(1,10)=311.2, p<$ $.001]$.

The group performance at each degree of desynchrony is shown in Figure 1. The solid line shows the performance measures of the present tests; for comparison, the dashed line shows the performance reported by Remez et al. (2008) when the degree of desynchrony varied unpredictably from trial to trial. The contrast between predictable desynchrony (square filled bullets, solid line) and unpredictable desynchrony (round open bullets, dashed line) is simple to describe. With unpredictable desynchrony, perceivers exhibited little tolerance of temporal distortion, and performance was uniformly poor except in three conditions: veridical ( 0 -msec desynchrony) and the two conditions of minimal desynchrony (50-msec lead, 50 -msec lag). Blocking the test items to exhibit consistent desynchrony affected performance only in these three conditions. There was no evidence that exposure to consistent desynchrony promoted better performance at more extreme values, and, accordingly, there is no evidence of a change in the span of desynchrony tolerance. The estimates of Fu and Galvin (2001) and Remez et al. (2008) are corroborated by these new measures.

\section{DISCUSSION}

The contrast in performance that was observed between predictable and unpredictable auditory desynchrony answers a practical question while posing a theoretical one. Practically, the measures reported here show that a temporally veridical sine-wave sentence actually loses 
a bit of its intelligibility if it occurs in a test block with sentences rendered unintelligible by desynchrony of one tone component. Whether this decrease in performance is attributed simply to the strangeness of sine-wave vocal timbre or is seen as a cost of uncertainty about unpredictably varying desynchrony, performance with small temporal distortion also exhibited the effect observed with temporally veridical items, although at lower absolute levels of performance. This shows that within the range of tolerable desynchrony, a consistent degree of temporal distortion occurring across different items in a test block offers a perceiver a better opportunity to resolve phonetic features from the time-varying properties of individual sentences. This propensity for improvement is unlikely to stem from the use of unintegrated auditory samples. However, an improvement in performance under conditions of consistent minimal temporal distortion seems to be the only adaptable aspect of the perceptual tolerance of desynchrony in auditory samples of speech, posing a theoretical question.

Why did performance fail at consistent desynchronies greater than $50 \mathrm{msec}$ ? A simple answer is that there remained no auditory property available to elicit phonetic impressions once sensory integration of the sine-wave components was lost. This can be attributed to the methods shared by the present study and the precedents of Remez et al. (2008) and Fu and Galvin (2001), in contrast to other techniques that permitted residual intelligibility in conditions in which sensory integration was arguably poor. When desynchrony was too great for integration to occur, intelligibility was simply lost.

More broadly, the difference in desynchrony tolerance in the integration of auditory components and the integration of audiovisual components is striking. Different methods have been applied to estimating the tolerance of temporal distortion in the sensory integration of auditory and visual samples. Moreover, varied measures have been applied to calibrate acuity in the perceptual resolution of auditory and visual coincidence (reviewed by Conrey \& Pisoni, 2006). From these, it is readily apparent that the temporal characteristics of unimodal auditory integration and bimodal audiovisual integration simply differ. Specifically, audiovisual integration appears to extend to $180 \mathrm{msec}$ of temporal offset when visual samples lead auditory samples. This modality-specific difference in the tolerance of temporal distortion is difficult to reconcile with the conceptualization that unanalyzed sensory samples of speech are functionally amodal until phonetic properties are resolved (Fowler, 2004; Green, 1998; Rosenblum, 2005).

Some recent studies have shown that the degree of temporal distortion that is tolerated in audiovisual integration is adaptable in the multimodal perception of speech (Navarra et al., 2005) and other events (Vroomen, Keetels, de Gelder, $\&$ Bertelson, 2004). Yet there is nothing in the present findings to encourage a conclusion that perceptual adjustment to consistent unimodal auditory distortion is especially likely or possible, and certainly not to the degree of tolerable temporal distortion demonstrated for audiovisual integration of audible and visible speech, which approaches $180 \mathrm{msec}$ (Bertelson, Vroomen, \& de Gelder, 1997; Munhall, Gribble, Sacco, \& Ward, 1996). On the basis of the results of the present project, it appears that sensory integration of auditory samples of speech is both fast and stable, and that this differs from the more forgiving and adaptable dynamic of multimodal integration. In order to understand the principles by which the window of temporal integration may be narrow and unwavering, as reported here, or wide and adaptable, as seen in the multimodal literature, new studies are needed to extend the inquiry.

\section{AUTHOR NOTE}

The authors are grateful for the advice and encouragement of Jennifer Pardo, Philip Rubin, Michael Studdert-Kennedy, and Emily F. Thomas. This research was supported by an award from the National Institute on Deafness and Other Communication Disorders (DC00308) to Barnard College. Correspondence concerning this article should be addressed to R. E. Remez, Department of Psychology, Barnard College, Columbia University, 3009 Broadway, New York, NY 10027-6598 (e-mail: remez@columbia.edu).

\section{REFERENCES}

Bernstein, L. E., Auer, E. T., Jr., \& Moore, J. K. (2004). Audiovisual speech binding: Convergence or association? In G. A. Calvert, C. Spence, \& B. E. Stein (Eds.), The handbook of multisensory processes (pp. 203-223). Cambridge, MA: MIT Press.

Bertelson, P., Vroomen, J., \& de Gelder, B. (1997). Auditoryvisual interaction in voice localization and in bimodal speech recognition: The effects of desynchronization. In C. Benoit \& R. Campbell (Eds.), Proceedings of the Workshop on Audio-Visual Speech Processing: Cognitive and computational approaches (pp. 97-100). Rhodes, Greece: ESCA.

Bregman, A. S. (1990). Auditory scene analysis: The perceptual organization of sound. Cambridge, MA: MIT Press.

Conrey, B., \& Pisoni, D. B. (2006). Auditory-visual speech perception and synchrony detection for speech and nonspeech signals. Journal of the Acoustical Society of America, 119, 4065-4073.

Darwin, C. J. (1997). Auditory grouping. Trends in Cognitive Sciences, 1, 327-333. doi:10.1016/S1364-6613(97)01097-8

EGAN, J. P. (1948). Articulation testing methods. Laryngoscope, 58, 955-991. doi:10.1288/00005537-194809000-00002

Elliott, T. M., \& Theunissen, F. E. (2009). The modulation transfer function for speech intelligibility. PLoS Computational Biology, 5, e1000302.

Fowler, C. A. (2004). Speech as a supramodal or amodal phenomenon. In G. A. Calvert, C. Spence, \& B. E. Stein (Eds.), The handbook of multisensory processes (pp. 189-201). Cambridge, MA: MIT Press.

Fu, Q.-J., \& GaLVIN, J. J., III (2001). Recognition of spectrally asynchronous speech by normal-hearing listeners and Nucleus-22 cochlear implant users. Journal of the Acoustical Society of America, 109, 1166-1172. doi:10.1121/1.1344158

GreEN, K. P. (1998). The use of auditory and visual information during phonetic processing: Implications for theories of speech perception. In R. Campbell, B. Dodd, \& D. Burnham (Eds.), Hearing by eye II: Advances in the psychology of speech reading and audiovisual speech (pp. 3-25). Hove, U.K.: Psychology Press.

Greenberg, S., \& AraI, T. (1998). Speech intelligibility is highly tolerant of cross-channel spectral asynchrony. Joint Proceedings of the Acoustical Society of America and the International Congress on Acoustics (pp. 2677-2678). Seattle: Acoustical Society of America.

Greenberg, S., Carvey, H., Hitchcock, L., \& Chang, S. (2003). Temporal properties of spontaneous speech: A syllable-centric perspective. Journal of Phonetics, 31, 465-485.

Kalikow, D. N., Stevens, K. N., \& Elliott, L. L. (1977). Development of a test of speech intelligibility in noise using sentence ma- 
terials with controlled word predictability. Journal of the Acoustical Society of America, 61, 1337-1351.

Massaro, D., \& Stork, D. (1998). Speech recognition and sensory integration. American Scientist, 86, 236-244.

McGurk, H., \& MACDonald, J. (1976). Hearing lips and seeing voices. Nature, 264, 746-748.

Munhall, K. G., Gribble, P., Sacco, L., \& Ward, M. (1996). Temporal constraints on the McGurk effect. Perception \& Psychophysics, $\mathbf{5 8}, 351-362$.

Navarra, J., Vatakis, A., Zampini, M., Soto-Faraco, S., HumPhreys, W., \& Spence, C. (2005). Exposure to asynchronous audiovisual speech extends the temporal window for audiovisual integration. Cognitive Brain Research, 25, 499-507. doi:10.1016/ j.cogbrainres.2005.07.009

PoEPPEL, D. (2003). The analysis of speech in different temporal integration windows: Cerebral lateralization as "asymmetric sampling in time." Speech Communication, 41, 245-255. doi:10.1016/S0167 -6393(02)00107-3

Remez, R. E., Ferro, D. F., Wissig, S. C., \& Landau, C. A. (2008). Asynchrony tolerance in the perceptual organization of speech. Psychonomic Bulletin \& Review, 15, 861-865. doi:10.3758/PBR .15.4.861

Remez, R. E., Rubin, P. E., Berns, S. M., Pardo, J. S., \& Lang, J. M.
(1994). On the perceptual organization of speech. Psychological Review, 101, 129-156. doi:10.1037/0033-295X.101.1.129

Remez, R. E., Rubin, P. E., Pisoni, D. B., \& Carrell, T. D. (1981). Speech perception without traditional speech cues. Science, 212, 947 950. doi:10.1126/science.7233191

Rosenblum, L. D. (2005). Primacy of multimodal speech perception. In D. B. Pisoni \& R. E. Remez (Eds.), The handbook of speech perception (pp. 51-78). Oxford: Blackwell.

RuBIN, P. E. (1980). Sinewave synthesis. Internal memorandum, Haskins Laboratories, New Haven, CT.

Saberi, K., \& Perrott, D. R. (1999). Cognitive restoration of reversed speech. Nature, 398, 760. doi:10.1038/19652

Silipo, R., Greenberg, S., \& AraI, T. (1999). Temporal constraints on speech intelligibility as deduced from exceedingly sparse spectral representations. In Proceedings of Eurospeech 1999 (pp. 2687-2690). Budapest: ESCA.

Sumby, W. H., \& Pollack, I. (1954). Visual contribution to speech intelligibility in noise. Journal of the Acoustical Society of America, 26, 212-215. doi:10.1121/1.1907309

Vroomen, J., Keetels, M., de Gelder, B., \& Bertelson, P. (2004). Recalibration of temporal order perception by exposure to audiovisual asynchrony. Cognitive Brain Research, 22, 32-35. doi:10.1016 j.cogbrainres.2004.07.003

\section{APPENDIX \\ The Sentences Used in the Experiment}

A pencil with black lead writes best.

Cut the meat into small chunks.

Football is a dangerous sport.

He ran halfway to the hardware store.

Her purse was full of useless trash.

His boss made him work like a slave.

Press the pants, and sew a button on the vest.

The bark of the pine tree was shiny and dark.

The beauty of the view stunned the young boy.

The bill was paid every third week.

The drowning man let out a yell.

The sandal has a broken strap.

The steady drip is worse than a drenching rain.

The watchdog gave a warning growl.

Two blue fish swam in the tank.

(Manuscript received April 14, 2010;

revision accepted for publication June 27, 2010.) 